\title{
INTERSUBJETIVIDAD, INTERCULTURALIDAD Y POLÍTICA
}

\author{
INTERSUBJECTIVITY, INTERCULTURALITY AND POLITICS
}

\author{
Javier San Martín ${ }^{1}$ \\ UNED (España)
}

Recibido: 30-11-2014

Aceptado: 02-02-2015

\begin{abstract}
Resumen: En el presente trabajo se trata de explicitar, desde la intersubjetividad propia de la fenomenología, el contenido de la interculturalidad. Para ello se elucida, en primer lugar, la relación de la fenomenología con la intersubjetividad. Se ve en segundo lugar en qué medida este punto se cruza con una noción histórica de la subjetividad hasta el punto de ser la historia consustancial a la subjetividad trascendental. Desde este punto de vista, la fenomenología implica una filosofía de la historia en la que se comprende la génesis y desarrollo de la filosofía desde el horizonte etnológico. Desde este pensamiento, la interculturalidad se muestra como el concepto que piensa la última etapa de nuestra historia $y$, pensada ahora desde las categorías de Kant sobre el lugar de la filosofía, se entiende como la creación de un espacio moral en el que somos responsables de los otros presentes y futuros como sujetos co-constituyentes del mundo. El artículo se apoya fundamentalmente en el desarrollo biográfico de estos temas
\end{abstract}

Palabras-clave: Intersubjetividad, horizonte etnológico, interculturalidad.

\begin{abstract}
The paper intends to develop the meaning of multiculturalism from the notion of intersubjectivity of the phenomenology. For doing it first of all the relation of the phenomenology and intersubjectivity is elucidated. Secondly it is explored to what extent this point crosses a historical notion of subjectivity, to the point that history is inherent to it. From this point of view, phenomenology involves a philosophy of history in which the genesis and development of philosophy is understood from the ethnological horizon. Then multiculturalism is shown as the concept that thinks the last stage of our history and thought now from Kant's categories about the position of philosophy, as the creation of a moral space in which we as co-constituent subjects of the world are responsible for present and future others. The paper is based primarily on the biographical development of these issues.

Key-words: Intersubjectivity, ethnological horizon, interculturality.

[1] (jsan@fsof.uned.es) Catedrático de Antropología Filosófica en la Universidad Nacional de Educación a Distancia. Ha realizado estancias de investigación en las Universidades de Lovaina y de Friburgo y ha impartido docencia en la Universidad de Buenos Aires y la Universidad Autónoma del Estado de México, entre otras. Javier San Martín ha centrado tanto su formación filosófica como sus publicaciones en tres ámbitos, la fenomenología, la antropología y la filosofía de Ortega.
\end{abstract}


Ante todo quiero agradecer a las organizadoras de nuestro Congreso esta invitación que me permite volver a Castellón, Universidad con la que bien saben que mantengo una estrecha vinculación desde los años 90, cuando empezamos con los seminarios de la SEFE, de los que mi querido amigo Vicent Martínez Guzmán era uno de los más asiduos participantes, acompañando a nuestro inolvidable Fernando Montero Moliner. Estoy hablando de fechas en las que más de uno de los aquí presentes visitabais aún la Escuela Primaria. Pues la primera sesión del seminario fue en diciembre de 1989. Veinticinco años hace ya de aquella fecha, y desde entones, cuando esta excelente Universidad era aún Colegio Universitario, no he dejado de colaborar en una u otra medida con aquel grupo de entusiastas amigos y compañeros. Varios de mis escritos han tenido como punto de partida algún evento organizado, bien por la Sociedad de Filosofia de Valencia, bien por el Departamento al que pertenecen Vicent Martínez Guzmán, Salvador Cabedo, Domingo García-Marzá, Sonia Reverte, o ya las dos organizadoras de este Congreso, Sonia París e Irene Comins. También participé varias veces en el master para la Paz, incluso fuera de España, pues di un curso en la sección de Toluca, ciudad con la que, por otras razones, mantengo un muy estrecho contacto.

El tema del que hoy quiero hablar es para mí muy querido, profundamente vinculado a mi visión de la filosofía y, en especial, de la fenomenología que inspira mi matriz filosófica, y muy vinculado también al modo como yo practico la fenomenología, a saber, como antropología filosófica. El trabajo va a ser desarrollado atendiendo a mi propio progreso profesional desvelando los diversos elementos que aparecen en el título. En el primer apartado, como punto de partida, comentaré la relación de la fenomenología con la intersubjetividad. En el segundo estudiaré la relación de la fenomenología con la historia, desde el concepto de historicidad de lo trascendental. El tercer apartado, el más largo, empezará ya a formular la problemática de la interculturalidad, partiendo de la relación de la filosofía con la ampliación del horizonte etnológico. Terminaré viendo el lugar en el que la interculturalidad tiene sentido para nosotros desde una perspectiva moral.

\section{Reducción, intersubjetividad y antropología}

La fenomenología incluye necesariamente la intersubjetividad. Lo que está en cuestión en ella es la antropología filosófica. Para la fenomenología, que se ve a sí misma como la filosofía depurada metodológicamente, la filosofía surge del contexto de la multiculturalidad. Justo este contexto obliga a redefinirse como antropología que debe ratificarse en la interculturalidad. Ese marco obliga a pensar una política acorde con esa interculturalidad.

Al ir cerrando la biografía de uno, después de tantos años en el trabajo universitario docente, se presentan dificultades a la hora de indicar en 
qué medida su trabajo ha evolucionado, se ha enriquecido o empobrecido, ha progresado o no lo ha hecho, qué conceptos o problemáticas son radicalmente nuevas en él. Digo esto porque yo hablo de estos temas consignados en el título desde lo que ahora se llama el "nuevo Husserl", que con ese nombre es algo reciente, pero que es el Husserl sobre el que escribí ya mi tesis doctoral presentada nada menos que en 1972. Muchos de los textos en que entonces basaba mi investigación son ahora reivindicados como la fundamentación de una nueva visión de Husserl. A partir de esos textos, algunos ahora ya publicados en el tomo XXXIV de Husserliana ${ }^{2}$, para mí siempre estuvo muy claro que tenía que revisar la relación de Husserl con la antropología. Las consecuencias de esa revisión ya las expuse a principios de los ochenta. Pero ahora ha adquirido perfiles más claros, sobre todo al tener que volver sobre esas tesis para definir mejor sus posibilidades, sobre todo porque el debate se ha acentuado en muchos más centros de investigación ${ }^{3}$.

En ese proceso hay que señalar como una fecha especialmente importante el año 1988, que coincidió con la fundación de la $\mathrm{SEFE}^{4}$, mi ida de año sabático a Alemania y la publicación de dos textos fundamentales para ratificar, profundizar y en cierta manera también reorientar mi propio trabajo. Los textos fueron la publicación del tomo XXVII de Husserliana, que incluye los artículos sobre Renovación del hombre y la cultura; y la esperada publicación de la VI Meditación cartesiana de Eugen Fink, que tuvo lugar a finales de ese año, por lo que me encontró en Alemania ${ }^{5}$.

[2] Uno de los textos básicos fue el manuscrito B I 5/IX, en el que Husserl plantea la superación del desasimiento o abstención de la posición del mundo, [die Enthaltung von der Setzung der Welt], lo que quiere decir que la epojé en el sentido usual queda superada, por lo que es inevitable un desplazamiento [Verschiebung] del sentido (ver Hua XXXIV, p. 60) de la epojé fenomenológica una vez practicada la reducción. Este manuscrito, que en su momento citara Gerd Brand en su tesis doctoral (como libro Welt, Ich und Zeit nach unveröffentlichten Manuscriten Edmund Husserls, Martinus Nijhoff, 1955), y que yo cito en mi tesis doctoral de 1972 y luego en la reelaboración de esta (La estructura del método fenomenológico, p. 185), actualmente se ha publicado, como la mayor parte del manuscrito B I 5, en Husserliana XXXIV, pp. 228-253.

[3] En nuestro mundo lingüístico, en Argentina, nuestra querida amiga Julia V. Iribarne, recientemente fallecida, que trabajara especialmente la problemática de la intersubjetividad, se interesó con profundidad en lo que ella llama antropología trascendental. También está teniendo el tema resonancias en México, en la Universidad del Estado de México, de Toluca, y en la Veracruzana, de Xalapa, en el primer caso, con la doctora Marcela Venebra, y en el segundo con el doctor Rubén Sánchez Núñez.

[4] Al final del Congreso Internacional de Fenomenología que organizamos en Santiago en colaboración con el Instituto dirigido por M. Teresa Tyminiecka.

[5] Fue una suerte el hecho de estar en Alemania en ese momento porque me permitió tener acceso a la VI Meditación cartesiana antes incluso de que fuera catalogada en la Universidad de Friburgo, con lo que ya antes de esas Navidades 1988/89 pude empezar a leer la importante contribución, en la que vería una ratificación de muchas de las interpretaciones con las que yo había operado hasta entonces.

THÉMATA. Revista de Filosofía, №52 julio-diciembre (2015) pp.: 129-145 doi: 10.12795/themata.2015.i52.07 
En el primero se habla de la función renovadora de la filosofía en relación con el ser humano, en un plano plenamente antropológico, no sólo teórico, sino principalmente práctico. En el texto de Fink se hace patente toda la problemática de la deshumanización que la metodología fenomenológica supone por la teoría del epojé, porque al abstenernos de la posición del mundo, quedo incluido ahí también yo mismo como ser humano, porque soy parte del mundo; pero a la vez se constata inmediatamente la necesidad de la vuelta a la humanidad, por tanto una rehumanización, si la fenomenología quiere operar o aparecer como ciencia, porque como ciencia está dirigida a los demás, y para ello debe "aparecer" en el mundo. Esta ida y vuelta es una de las características fundamentales de la fenomenología plenamente aclarada y fundamentada por Eugen Fink ${ }^{6}$. La vuelta la convierte de modo necesario en una antropología filosófica porque todo lo que la fenomenología dice lo dice sobre nosotros, los humanos, aunque sea tomando al ser humano aclarado por la fenomenología, es decir, un humano que es más que humano, entendido éste en el sentido en que lo toman las ciencias naturales y sociales.

Pues bien, el tema o concepto filosófico que está en el centro de estos puntos y de esa metodología, de esas idas y vueltas, no es otro que el tema de la intersubjetividad, un tema que está en íntima conexión con los avatares de la fenomenología como ciencia. El problema viene de los efectos del método de la epojé, que parece dejarnos inermes a ese respecto, porque en su versión de Ideas I, la más usual, los otros desaparecerían. Ahora bien, la intersubjetividad es imprescindible para definir el concepto de razón, que si por algo se caracteriza es por aludirse en ella a cualquier ser racional, porque lo legitimado racionalmente vale para todo sujeto racional. En la medida en que el objetivo de la fenomenología es convertirse en un saber riguroso, por tanto racionalmente legitimado, los otros están constitutivamente implicados en ella en cuanto ciencia, lo que no es fácil de compaginar con el sentido de la epojé de Ideas $I$.

Pero independientemente de lo que pudiera representar la epojé de Ideas I, en efecto, el tema de la intersubjetividad es un tema nuclear de la fenomenología prácticamente desde el principio. Yo tuve suerte en mi tesis doctoral. De hecho yo me pasé de Merleau-Ponty, que es a quien había empezado a estudiar, a Husserl, porque la reducción trascendental, de la que se hablaba en el prólogo de la Fenomenología de la percepción, me resultaba ininteligible al tener que conciliar elementos opuestos: el otro que abandono en la epojé, con el otro sin el que no doy un paso porque la ciencia y la razón implican a los otros. Este fue el dilema que me llevó a estudiar la reducción

[6] Este fue el tema de mi curso en Buenos Aires en 1992, que luego se publicó con el título Fenomenología y Antropología. La mala suerte quiso que la editorial Almagesto, en que salió el libro en 1997, quebrara ese mismo año, con lo que el libro quedó prácticamente sin distribución.

THÉMATA. Revista de Filosofía, №52 julio-diciembre (2015) pp.: 129-145 doi: 10.12795/themata.2015.i52.07 
fenomenológica, entendida desde la epojé, porque en ella parecía estar el problema radical de todo.

Como ese fue el tema con el que entré en la fenomenología de Husserl, ya en la tesis descubrí las obligadas idas y vueltas que confluían principalmente en la necesidad de redefinir el sentido de la epojé y la reducción. Pero a la vez todo ello me llevaba a reconfigurar el horizonte antropológico de la fenomenología, permitiéndome diseñar una antropología filosófica que vivía del horizonte marcado por la fenomenología trascendental, que me llevaba a poner como lema de cualquier antropología filosófica la frase de Merleau-Ponty, de que:

No soy el resultado o encrucijada de las múltiples causalidades que determinan mi cuerpo o mi "psiquismo"; no puedo pensarme como una parte del mundo, como el simple objeto de la biología, de la psicología y de la sociología, ni cerrar sobre mí el universo de la ciencia. (Merleau-Ponty, 1945, II).

En ella se encierra una reivindicación con la que creo que debe empezar toda antropología filosófica, que, elaborada desde un horizonte fenomenológico, se convierte en filosofía primera porque las ciencias naturales y sociales han puesto todo su empeño, lo siguen poniendo y lo pondrán aún más, en "cerrar sobre nosotros el universo de la ciencia", es decir, en explicarnos totalmente, convirtiendo la filosofía en superflua y clausurando, así, todo espacio filosófico.. Hay que decir que la inmensa mayoría de los científicos que no tengan compromisos religiosos aceptarían que la ciencia conseguirá en su momento explicarnos totalmente, es decir, "cerrar sobre nosotros el universo de la ciencia". No es muy distinta la actitud de la antropología cultural respecto a la filosofía. Es lo terminó llevándome a una fórmula que se ha ido convirtiendo en un punto fundamental de mi concepción sobre qué representa la posición de la antropología cultural en relación con la filosofía, a saber, que en la antropología cultural se producía la "muerte antropológica de la filosofía", pues para la antropología cultural la filosofía no es sino un discurso de las sociedades occidentales sobre los otros sin más valor que el de otro relato cualquiera, sea quechua, trobriandés, nambikwara, dogón o lo que se quiera ${ }^{7}$. Así, era fundamental recuperar una filosofía que de entrada asumiera un sujeto intersubjetivo, que teóricamente debería poderse instanciar en cualquier cultura.

[7] A esa frase ya a principios de los ochenta añadí la lógica consecuencia: que la muerte antropológica de la filosofía llevaba la muerte filosófica de la antropología, porque el relativismo cultural en que se basa la muerte antropológica de la filosofía aniquila también a la propia antropología cultural como ciencia. Ver Para una superación del relativismo cultural, donde hablo de que "la reducción antropológica de la filosofía es la reducción filosófica de la antropología". San Martín, 2009, 38 y 130. Este texto proviene prácticamente en su totalidad de la memoria con que concursé a una Cátedra de Antropología para la Facultad de Filosofía de la Universidad Complutense, que la sacó el profesor de antropología cultural, José Luis García García. Ver J. San Martín Ciencia y Filosofía del hombre, tomo primero, pp. 131 ss. 1981 (inédito).

THÉMATA. Revista de Filosofía, №52 julio-diciembre (2015) pp.: 129-145 doi: 10.12795/themata.2015.i52.07 


\section{La historicidad de lo trascendental}

El siguiente punto era el relativo a la historia. Y en la historia se mezclan varias facetas. Incluidas de modo ineludible la antropología y la intersubjetividad en el seno de la fenomenología, se me planteaban varios puntos de interés. Primero, la humanidad es un sujeto trascendental porque el mundo está organizado desde cada uno de nosotros, aunque seamos muchos. Segundo, la subjetividad trascendental tiene una génesis, un desarrollo. El propio Husserl lo daba por obvio en el concepto de pasividad secundaria, por el cual la subjetividad asume los resultados de cada acto, que se convierten en matrices para los actos posteriores. La teoría de la habitualidad, que se hace patente cuando Husserl, ya en Ideas II, convierte al yo puro de Ideas I en sujeto de hábitos, pasa a ser un elemento básico de un desarrollo histórico, tanto en el terreno individual, donde es obvio, como en el terreno social, pues la cultura no es sino el repertorio de los usos o hábitos de una sociedad. En el terreno individual, cada una de esas matrices o hábitos son adquisiciones que actúan, una vez adquiridas, de modo pasivo, previo a todo acto posterior, como hábitos de reconocimiento, evaluación y acción. Desde ese momento, la subjetividad trascendental es histórica, porque además esos hábitos están previamente instaurados en la sociedad. Es la cultura la que nos sirve esos hábitos, y la cultura es un jardín de la diversidad.

Todo esto me planteaba a mí un problema al que, sin embargo, tardé en poner nombre, aunque terminé por formularlo antes de ir a Alemania en 1988, definiéndolo como la historicidad de lo trascendental. Si la trascendentalidad según Husserl admitía un enriquecimiento, la noción husserliana de trascendental estaba lejos de la kantiana, que parecía estar totalmente configurada desde el principio, por lo que no sería histórica. Además, si la transcendentalidad tenía una génesis desde la infancia, la historicidad era una de sus características constitutivas. La historicidad de lo trascendental fue una de las convicciones a que llegué antes de ir a Alemania en 1988, por tanto antes de mi lectura de la Sexta meditación cartesiana, por lo que la lectura de esta me resultó absolutamente fascinante, ya que en ella se da la razón del porqué de esa historicidad ${ }^{8}$. Más aún, si ya nos metemos en el terreno antropológico, cabía la pregunta de si no habría diversas etapas en la configuración de lo trascendental, un sujeto trascendental infantil, uno maduro, uno centrado en las cosas de su vida, otro batallando por una humanidad mejor, es decir, una transcendentalidad crítica.

[8] Abordé el tema en la siguiente comunicación en el Coloquio en Cerisy-la Salle, que organizaron Marc Richir y Natalie Depraz en 1994 cuyas actas se publicaron después. Ver del autor «La philosophie de l'histoire chez Fink et Husserl ", en Eugen Fink. Actes du Colloque de Cerisy-laSalle 23-30 juillet 1994, Rodopi Elementa, pp. 231-244, 1997.

THÉMATA. Revista de Filosofía, N52 julio-diciembre (2015) pp.: 129-145 doi: 10.12795/themata.2015.i52.07 
Con estos conceptos podía abordar la historia de la humanidad desde la mera hominidad, que podía ser propia de nuestros ancestros homínidos, hasta nuestros coetáneos preestatales, humanos plenos pero que no vivían en etapas en las que se diseñaran horizontes de plenitud humana; y nuestros coetáneos históricos, humanos de la era filosófica.

\section{De la intersubjetividad a la interculturalidad: el horizonte etnológico}

Una vez que hemos llegado a este punto, la fenomenología acogiendo en su filosofia de la historia la diversidad de los coetáneos, ya podemos pasar al nuevo concepto que se fragua para pensar ese momento, concepto que, a pesar de ser reciente, tenemos que reconocer que nos ha inundado pero que tiene un origen preciso en el tiempo, con la precisión un poco dilatada que puede ser propia de la pregunta de cuándo empezamos a hablar de algo. ¿Cuándo empezamos a hablar de interculturalidad? Desde luego, en nuestro mundo no hace tanto. Y aquí sí tuve yo una ventaja profesional, al tener que impartir, en la Universidad de Santiago de Compostela, cursos de Antropología cultural desde 1974 a 1979, y tener que lidiar desde la antropología cultural con el tema del relativismo cultural, que es lo que me permitió entender el nacimiento de la filosofía desde la complejidad del horizonte etnológico. Sin una clara familiaridad con la antropología cultural tal vez nunca hubiera llegado a comprender esta cuestión central ${ }^{9}$.

De lo que acabo de decir se infiere que el tema de la interculturalidad no estaba en mi primera etapa de trabajo. Sí estaba el tema de la intersubjetividad, y desde la antropología cultural estaba también el tema de la relación del horizonte etnológico con la filosofía. El tema de la interculturalidad ha ido ascendiendo en el horizonte temático a medida que la multiculturalidad y el multiculturalismo se adueñaban de la sociedad en términos cada vez más ineludibles, obligándonos a volver a pensar en términos muy drásticos el carácter de nuestra filosofía. Y hay que decir que esto aún está en marcha, o sea, no hemos terminado de asumir que la filosofía debe pasar el filtro de la interculturalidad. Baste con un ejemplo. Recientemente (febrero de 2014) ${ }^{10}$, tuve la

[9] Es lo que pienso que le ocurrió a José Gaos con La crisis de las ciencias europeas. A pesar de estar en México, país en el que la diversidad étnica es abrumadora, no parece haber captado la problemática antropológica de ese libro, toda la problemática del mundo de la vida, que es común y diferente, y que da un marco para resolver el problema del relativismo cultural. En cierta ocasión se lo hice ver a Juan Nuño, en una visita que hizo a España a mitades de los ochenta. La forma en que Gaos leyó La crisis fue con cierta irritación al ver que discípulos suyos como Luis Villoro ponían en ese libro ciertas esperanzas. Sobre La crisis como una filosofía de las ciencias humanas, ver la tercera parte de Fenomenología y antropología, San Martín 1997

[10] Esta conferencia, en una sesión compartida con Vicent Martínez Guzmán, se leyó en mayo de 2014 .

THÉMATA. Revista de Filosofía, №52 julio-diciembre (2015) pp.: 129-145 doi: 10.12795/themata.2015.i52.07 
oportunidad de participar en el tribunal de una tesis sobre el último Vattimo, el que desarrolla una filosofía cristiana desde el concepto paulino de kénosis, el rebajamiento o vaciamiento de Dios en la Encarnación al devenir hombre. Es un modelo de filosofía, que puede resultar sumamente atractiva, pero que resulta nada intercultural, porque la kénosis es un concepto estrictamente cristiano que solo vale para los que aceptan esta religión, cuyo número obviamente es muy amplio, pero aun así no llega a agrupar ni a un veinte por ciento de la humanidad. Pero, si la filosofía ha de cumplir lo de ser funcionarios de la humanidad, no puede ni debe excluir a ningún humano.

Voy a intentar hacer ver cómo y en qué medida el concepto de intersubjetividad en la fenomenología y en la antropología filosófica se convierte en interculturalidad.

La filosofía se inicia cuando en un momento de la historia el saber científico está suficientemente desarrollado pero hay discrepancias claras respecto a las respuestas que se dan sobre el sentido de la vida humana del que dependen las grandes orientaciones prácticas de la vida individual y social, lo que ocurre por la presencia simultánea de muchas soluciones diferentes, que no pueden ser verdaderas a la vez. Es, exactamente, lo que decía Jenófanes de Colofón, que para los etíopes los dioses son "chatos y negros", mientras que para los tracios son de "ojos azules y rubios" (Kirk y Raven 1969, 241, fr. 16). De la diferente concepción de los dioses se derivan todas las diferencias morales y políticas con que estamos habituados a convivir. Jesús Díaz resume muy bien este punto, tomando como referencia pensadores de la "pasada centuria", entre ellos a Klaus Held. El resumen es que para todos esos filósofos "el genio griego residió en haberse hecho cargo por primera vez de modo explícito, y mediante un discurso articulado que tuvo continuidad en el tiempo, del problema que representaba la pluralidad de concepciones del bien, la justicia y la verdad" $(2012,110)$. Y justo esto ocurrió, no en el centro de Grecia, sino en los lugares donde la multiculturalidad era obligada, como son las colonias griegas. En nuestro caso lo tenemos muy claro porque la filosofía se inicia en las colonias griegas de Asia Menor, Mileto y Éfeso, y en el sur de la península italiana, Elea.

No ha sido frecuente tomar nota de este hecho. Se ha enmascarado el comienzo de la filosofía con actitudes psicológicas, el asombro; o sociológicas, la isegoría, el derecho a un uso igual de la palabra, consecuente, en gran medida, con las guerras y en el abandono de las aldeas, generando un demos. Creo que ninguna explica por qué de repente los griegos, u otros pueblos como los chinos o indios (Mall y Hülsmann 1989), se ponen a hacerse preguntas sobre el mundo no respondidas en los mitos. La razón es que la multiculturalidad real ya ha debilitado los mitos por la sencilla razón de que en esa situación multicultural coinciden mitos procedentes de varias culturas. 
La filosofía es hija de la ampliación del horizonte etnológico. Entiendo por horizonte etnológico el horizonte cultural-mitológico propio y particular de un pueblo. La ampliación de ese horizonte es por la presencia de otros mitos o relatos fundacionales. Es lo que se dio en las colonias griegas. Y, luego, lo que se dio también con el descubrimiento de América, que llevó de nuevo a repensar la filosofía desde sus cimientos, dando lugar a la Filosofía Moderna, que sólo se entiende desde el trasfondo de la enorme ampliación del horizonte etnológico, que provoca el debate antropológico, en el que se pregunta qué somos, si tenemos algo común a priori que nos unifique o si somos meros resultado de las circunstancias, sean estas históricas, culturales o sociales. Es, por otro lado, el debate que se plantea también en la sofística, que no es sino la respuesta a la pregunta de si se puede aprender todo, por tanto, si la argumentación retórica, al margen de la verdad, sirve para lo uno y lo contrario. O si hay unas normas a priori a las que podamos llegar racionalmente y que son las que inspiran de una manera u otra, antes o después, todo sistema cultural.

Precisamente esta visión de la filosofía como una respuesta a la multiculturalidad, tema del que habla Husserl en uno de sus últimos textos ${ }^{11}$, nos lleva a un nuevo modo de pensar la relación de la filosofía con las ciencias humanas. Porque son dos —o tres, si queremos, pues podríamos incluir aquí la filología- las ciencias humanas que nos dan las diferencias culturales de los pueblos, la historia y la etnología o antropología cultural. Y es en este contexto en el que hay que pensar la interculturalidad.

Por otro lado, he hablado de dos ampliaciones del horizonte etnológico, la que provocó el origen mismo de la filosofía, y la que provocó el desarrollo y contenido de la filosofía moderna. Pues tenemos que hablar de una nueva ampliación del horizonte etnológico, la proveniente de la descolonización y consiguiente asunción de decenas y decenas de nuevos países en el espacio público y político común, que ha llevado a la migración de gentes de un país al otro y, ya a finales del siglo XX, a una nueva situación, que se intenta comprender con una nueva palabra, de origen tal vez diverso, pero cuyo sentido más preciso es el tener detrás esta nueva multiculturalidad, y que es la posmodernidad. La postmodernidad es el resultado de todo lo ocurrido ideológica, económica y, por fin, políticamente en la segunda mitad del siglo XX, de lo que se toma conciencia a finales del siglo, y que a principios de este ya es uno de los grandes temas para la reflexión filosófica: el multiculturalismo, porque la multiculturalidad, en nuevas condiciones políticas, se convierte en multiculturalismo.

[11] A comentar este texto de Hua XXIX, pp. 362-419 estuvo dedicada mi comunicación en el Congreso de la REF, de septiembre de 2014, actualmente en actualmente en Actas del I Congreso internacional de la Red española de Filosofía. Volumen II. Sección temática 1. Antropología filosófica y teoría de la cultura, 2015, pp. 71-82, y que lleva por título "La interculturalidad como génesis de la filosofía. Comentario al antepenúltimo texto de Husserl”.

THÉMATA. Revista de Filosofía, №52 julio-diciembre (2015) pp.: 129-145 doi: 10.12795/themata.2015.i52.07 
Así la nueva ampliación del horizonte etnológico, que tiene detrás la emergencia de nuevos países, con gran variedad de tamaños y presencia internacional, y la emigración, con la consiguiente masiva mezcla de culturas, en contextos políticos muy nuevos, ha generado la necesidad de responder al multiculturalismo con una nueva oferta, la interculturalidad. Este es el contexto filosófico, cultural y político de la interculturalidad que, pensada seguramente ya mucho antes, se ha convertido en elemento focal en los últimos años debido a que es la etapa de la planetarización o globalización ${ }^{12}$ la que acoge en su seno o provoca una creciente e imparable multiculturalidad o multietnicidad.

Llegados aquí, para abordar el sentido de la interculturalidad podríamos tomar como punto de partida los tres momentos en que la filosofía surge o es reformada, para ver qué ocurre con aquella multiculturalidad frente a la que se define. Podríamos tomar como referencia las ampliaciones del horizonte etnológico que acabamos de ver. Así, en el primer periodo, es muy posible que la filosofía tratara de ofrecer un discurso mediador con los otros, sólo que esos otros estaban muy metidos en el mito y la filosofía pretendía superar el mito. Pero pienso que tanto Platón, que utiliza el mito para enseñarnos, como Aristóteles buscan un discurso que conviniera a todos. Quizás porque tomando la frase de Jenófanes como modelo, los dioses no pueden ser ni negros y chatos, ni de ojos azules y rubios, porque los predicados que se les apliquen no les podrían advenir de ninguna cultura ${ }^{13}$. Es posible que desde Aristóteles se pueda entender mejor que desde otros autores la naturaleza de lo divino, e incluso que en gran medida su teoría de la virtud sea un buen modelo de interculturalidad. Así se podría entender el texto de Marta Nussbaum defendiendo el esencialismo de Aristóteles (Nussbaum, 1992; González Esteban, 1993). De hecho, es interesante, por ejemplo, que entendieran al humano como participante de la divinidad por el hecho de sus competencias cognitivas, una cuestión que ha sido muy frecuente en muchas culturas.

Más nos sirve la consideración de la diferencia cultural en la segunda etapa. En ella el acceso a las otras culturas fue fundamentalmente por la conquista y consiguiente consideración de la superioridad de la occidental, incluyendo muchas veces en ese proceso la aniquilación de los culturas de los otros.

[12] Sobre qué representa esta etapa, desde la fenomenología, ver del autor "La planetarización o globalización, nueva dimensión de la multiculturalidad”, en López/Díaz, 2012, pp. 233-268.

[13] Jenófanes había visto muy bien la dependencia de los dioses respecto de la cultura y el momento, es decir, su relatividad cultural, siendo así que eso parecía incompatible con la noción de Dios. Así, en el fragmento 11 dice que Homero y Hesíodo atribuían a los dioses cuantas cosas constituyen "vergüenza y reproche entre los hombre". En el 14, toma nota de que los mortales se imaginan "que los dioses han nacido y que tienen vestidos, voz y figura humana como ellos." El más interesante es el 15, donde dice que si los bueyes, caballos o leones tuvieran manos y fueran capaces de pintar, pintarían a los dioses, los caballos como caballos, lo bueyes como tales y los leones también como ellos mismos. Es una descripción magnífica del etnocentrismo y del relativismo de las creencias (Kirk y Raven, 240 s.)

THÉMATA. Revista de Filosofía, N52 julio-diciembre (2015) pp.: 129-145 doi: 10.12795/themata.2015.i52.07 
De todos modos, es el momento en el que empieza el estudio de los otros aunque fuera poniéndolos como inferiores. En esa posición se escondía el tema del debate sobre la amplitud del carácter humano, aunque las diferencias fueran consideradas en principio como diferencias en el tiempo: nosotros, los europeos, representantes o herederos del pueblo elegido por Dios para la redención, tenemos a los otros como nuestros primitivos, como éramos nosotros cuando teníamos su tecnología, pero, en todo caso, los otros también son otros. Por eso la antropología es ciencia de todos (ver San Martín 2009, 150).

En este estadio, el de la filosofía moderna, se diseñó la antropología cultural, como un saber de los otros, como un saber de las diferencias, pero ella misma estuvo sometida a tres movimientos en relación con las diferencias. Primero, comprende las diferencias como etapas del desarrollo hacia una plenitud a la que nosotros habríamos llegado o que habríamos alcanzado. Segundo, las concebirá en un plano de igualdad, desde un relativismo cultural en el que todas las diferencias parecen iguales; pero, en realidad, el relativismo cultural vivía en una contradicción, porque, bajo el manto de la igualdad del reconocimiento, se daba el hecho de que los diferentes estaban en el Tercer Mundo, muchas veces aún colonizados y sin capacidad de decisión, que vivían muchas veces en reservas o territorios sin verdadera capacidad política; y si ya no eran miembros de colonias o reservas, eran ciudadanos de estados empobrecidos. Precisamente en virtud del relativismo cultural, teóricamente nadie podría hacer ciencia, pero de hecho quienes hacían la ciencia antropológica eran los científicos del primer mundo. Los otros tenían aparentemente una cultura propia tan digna como la de los antropólogos, pero eran estos los que hacían de hecho antropología. Por eso no había aún verdadera interculturalidad.

Para la interculturalidad tenía que haber un cambio radical, que no pasa por el relativismo cultural, sino, primero, por una radical descolonización. Segundo, por el reconocimiento de las diferencias en el pleno derecho tanto de ellas como de los grupos humanos que configuran. Tercero, y es lo más importante, por la diferenciación entre nosotros de elementos étnicos particulares y elementos no étnicos, pensables estos desde cualquier cultura. Así pues, ahora entramos ya en un nuevo terreno, el del reconocimiento de la construcción de las otras culturas como la propia, con la distinción en todo sistema de elementos étnicos particulares y elementos no étnicos comunes ${ }^{14}$.

Por eso hizo falta el tercer estadio, el de la descolonización para poder considerar las diferencias culturales como diferencias tan plenas como nuestras peculiaridades. Este movimiento, que es propio del siglo XX, tiene muchos matices y reflejos dentro de la propia fenomenología. Esta se encuentra en una situación en que las ciencias humanas, sobre todo la antropología cultural, ya

[14] He comentado este punto en varios lugares, el primero en todo caso en mi primer libro de antropología, La antropología, ciencia, ciencia humana, ciencia crítica, Montesinos, Barcelona, 1985. $3^{\text {a }}$ ed. 2001.

THÉMATA. Revista de Filosofía, №52 julio-diciembre (2015) pp.: 129-145 doi: 10.12795/themata.2015.152.07 
nos ha mostrado las diferencias y lo que es el mundo de la vida en que cada comunidad vive, incluida la nuestra. Nosotros vivimos tan en nuestro particular mundo de la vida como cualquier otro. La fenomenología distingue dos grandes épocas históricas, la prefilosófica y la filosófica; y, en esta, la antigua y la moderna. En la última, el mundo de la vida termina asumiendo las explicaciones científicas. La inmensa mayoría de los pueblos, antes de la segunda mitad del siglo, vivía en mundos precientíficos. En ellos, la antropología cultural, nos ofrece el acceso a sus diferencias.

Husserl se interesó por los estudios de Lévy-Bruhl porque nos anunciaban la posibilidad de acceder a esas diferencias y entender hasta qué punto esas diferencias constituían los mundos particulares en que los otros vivían. Incluso nos servía para comprender cómo esos mundos son resultados de la acción social de esos pueblos, dándose así una visión más clara de la forma en que la fenomenología considera nuestra relación con el mundo, como un mundo que es sedimentación de nuestras operaciones de fundación del sentido. Cada mundo es, así, particular, pero eso no quiere decir que no haya una comunicación posible ente ellos. Más bien lo contrario, la filosofía es capaz de descubrir, detrás de lo diferente, de lo étnico, lo no étnico común a todos los humanos (San Martín 2006). Desde esta plataforma, que ya diseñara Husserl en La crisis de las ciencias europeas, debemos abordar la interculturalidad en la actualidad.

Dicho todo esto, tenemos que volver a la intersubjetividad. Como ya lo hemos afirmado, la fenomenología se plantea desde el principio en el terreno de la intersubjetividad. Su apuesta por la razón, que es el único medio cognoscitivo permitido a la filosofía - a diferencia de las respuestas propias de la tradición, amparadas generalmente en relatos míticos-, supone pensarse siempre en la experiencia del otro, de modo intersubjetivo, porque sólo con los otros es viable el concepto de razón. La intersubjetividad es la palabra con la que denominamos la experiencia del otro y el hecho de estar de siempre referidos a los otros. Mas los otros son otros cercanos, menos cercanos y alejados.

En los otros, como en mí, hay varios planos, está el cuerpo y su movilidad, gestualidad y expresión. Y está el plano de la cultura, es decir, aquellas acciones que se emprenden en la seguridad de que son comunes al grupo, por lo que se conoce la participación de los otros en esas acciones, que remiten a todo un campo cultural, en el cual también yo estoy inmerso. Conocer al otro es conocer todos estos niveles. Y la intersubjetividad funciona en círculos de familiaridad y cercanía, pero también está constituida por los círculos de los más lejanos. Husserl ensalza que Lévy-Bruhl nos haya enseñado a penetrar en el alma de los más alejados (Husserl 1995), es decir, de poder entrar en el alma de personas sumamente divergentes de nuestra cultura. No es importante si Lévy-Bruhl podía estar o no equivocado en sus apreciaciones sobre la 
mentalidad de los "primitivos"15. Lo que nos dice Husserl es que cada pueblo tiene su cultura, su mundo, su apriori, y en este todo encaja. Pero también, que podemos acceder a ese mundo, más aún, que para la fenomenología tienen sus ventajas esos pueblos porque en ellos se ve muy bien la correspondencia entre el mundo y su sociedad. Es algo así como decir que en esos pueblos sencillos se observa mejor el a priori de correlación, en el que hay que meter también la correlación entre los sistemas ideológicos, el mundo social y el mundo económico. Todo se ve de modo más conjuntado que en los pueblos con Estado, en los que los diversos niveles pueden tener orígenes profundamente diversos y por ello haber profundas divergencias entre los mismos.

Estos temas de Husserl nos ponen en un terreno de cómo podemos conocer a los otros, lo que es una cuestión propia, tanto del intercambio ordinario entre las personas de distintas culturas, cuestión ahora importante en un mundo multicultural, como del procedimiento propio de las ciencias humanas, con las diversas finalidades de estas, bien sea contribuir de modo decisivo y seguro al punto anterior, por ejemplo, si se quiere proceder de modo seguro en un programa de ayuda social en un ámbito multicultural; o ya desde una perspectiva científica, por el interés de conocer más allá de las primeras manifestaciones de las diferencias, al entender que los elementos culturales podrían ser como los signos de una lengua, elementos que se diferencian por la posición en una estructura, como ocurre con los elementos del habla. En ese caso el antropólogo puede tratar de conocer las diferencias como manifestaciones o variaciones de un campo estructural cuya lógica pretende descubrir. Ese es el modo como el estructuralismo se acerca a las diferencias con su metodología. En ese terreno se sitúa la disputa de Merleau-Ponty con Levi-Strauss (López 2012), al decirle aquel que de ese modo, propiamente hablando, no conoce a los otros, ya que se le escurrirían de las manos, porque la estructura sin significados para los miembros de una cultura no es nada. Ese es también el terreno en el que Husserl plantearía el conocimiento del otro. Por eso, Husserl plantea el tema de la intersubjetividad, pero no el de la interculturalidad, aunque de todo lo dicho sí podemos sacar unas pautas para ver el terreno en el que se da la interculturalidad, que creo que es un paso, al menos, más allá de la fenomenología husserliana.

[15] Como se sabe, esa diferencia era un resto de la ideología que impregnaba el paradigma evolucionista (del evolucionismo cultural, que es anterior al evolucionismo biológico), teoría, por la época de Levy-Bruhl, muy cuestionada, hasta el punto de que Franz Boas ya había escrito uno de sus artículos más importantes contra ese presupuesto de la antropología usual aún en muchos países. Ver de Boas, The Mind of Primitive Man, (1911) en cuyo capítulo XI (pp. 201-227) se recoge y se desarrolla el argumento principal del libro, con el título "La mentalidad del hombre primitivo y el progreso de la cultura”, que amplía un artículo de 1901, que da título al libro entero.

THÉMATA. Revista de Filosofía, №52 julio-diciembre (2015) pp.: 129-145 doi: 10.12795/themata.2015.i52.07 


\section{Interculturalidad, filosofía y orden moral}

Yo he abordado este tema en relación con la posibilidad de dar un contenido al ideal husserliano de un orden moral del mundo. Este concepto lo toma Husserl de Fichte, y se remite a él tanto en las conferencias sobre el ideal del ser humano en Fichte, de 1917, como en los artículos sobre Kaizo, de 1922/24 ${ }^{16}$. En el orden moral del mundo, empezaríamos por reconocer a las personas plenitud en todos los sentidos, lo que está en relación con la corresponsabilidad que el otro tiene en la constitución del mundo. La filosofía empieza poniendo al otro como co-constitutivo, esa posición es la posición de otro idéntico a mí ${ }^{17}$. Una vez propuesto ese punto, las diferencias culturales me obligan a no dar por clausurada ninguna propuesta mía sin mediarla con otras culturas.

Y aquí tendríamos que hacer de nuevo distinciones, porque la interculturalidad no se debe referir al plano técnico, en el que reina la eficacia, todo aquello a lo que Kant se refiere con la palabra Geschicklichkeit, la competencia o la capacidad de llevar a cabo alguna acción eficaz, por ejemplo, para construir algún producto, digamos, hacer relojes, construir centrales nucleares o barcos. Ni tampoco al plano de preferencias prácticas, las que se refieren al orden social, que Kant entendería abarcadas o reguladas por la palabra Klugheit, que yo prefiero traducir por sagacidad o perspicacia, más que por prudencia, dado el carácter moral que esta implica. En el ejemplo de Kant, dado que uno ya sabe hacer relojes, el siguiente paso es ser capaz de venderlos, por tanto debe ajustar los relojes que hace a la capacidad de compra o a los gustos de aquellos a quienes se va a dirigir. Esto se consigue con la sagacidad o perspicacia, por las cuales debe conocer la situación efectiva de los otros. La antropología cultural, como una especie de antropología pragmática en el sentido de $\mathrm{Kant}^{18}$, se desenvuelve en este nivel, para enseñarnos a comportarnos entre los humanos; para ello debo, ante todo, conocer su cultura. La interculturalidad no plantea problemas especiales en este terreno, y la mejor prueba es que el comercio, por tanto el intercambio de productos, sea mediante el trueque, sea por mediación de la moneda, desde hace miles de años ha funcionado en el mundo al margen de los problemas de la

[16] Estos dos textos de Husserl fueron conocidos el año 1987 y 1989. En ellos se ve la orientación práctica de la filosofía. De los artículos sobre Kaizo me interesa desatacar la filosofía de las profesiones como regulación de la vida que se explicita en el tercer artículo. Ver del autor Teoría de la cultura, cap. IV, y Antropología filosófica II. Vida humana, persona y cultura, cap. XII.

[17] Los temas relacionados con la intersubjetividad y su relación con la ética constituyen el núcleo de la aportación de Julia V. Iribarne a la fenomenología. Sobre la teoría de la intersubjetividad es esencial su libro La intersubjetividad en Husserl. Bosquejo de una teoría (Iribarne 1987). Sobe la relación de la intersubjetividad y la ética ver Iribarne 2007, donde también hay un capítulo dedicado a los artículos sobre Kaizo.

[18] Sobre la posición de Kant en relación con la antropología y estos tres tipos de actitudes, ver San Martín 2013, 184 ss.

THÉMATA. Revista de Filosofía, N52 julio-diciembre (2015) pp.: 129-145 doi: 10.12795/themata.2015.i52.07 
interculturalidad. Los problemas vienen cuando consideramos los elementos que rigen el sentido de la vida, la estima de las personas, o, como decía Jesús Díaz (ver supra), las concepciones del bien, de la justicia y de la verdad. En el caso del ejemplo de Kant, no me basta con ser competente para hace relojes; de ser perspicaz y sagaz para vendérselos, sino de saber si, en unas condiciones económicas determinadas, conviene a esa sociedad gastarse el dinero en relojes. Si ponemos como ejemplo la construcción de centrales nucleares, se verá la necesidad de distinguir los tres niveles. Porque dado que se es competente para hacerlas; que se ha convencido a los vecinos de la utilidad y bienes que conseguirán con la electricidad producida nuclearmente, siempre viene después una decisión sobre la oportunidad político-moral de esa decisión. Es política porque hay que decidir dedicar muchos fondos que que podrían dedicarse a otros fines de la sociedad, por tanto hay que decidir el tema políticamente. Y es moral respecto a los presentes, pero, aun en el caso de una seguridad garantizada en su funcionamiento actual, nos cuestiona moralmente sobre todo respecto a las generaciones futuras a las que cargamos con unos residuos con los que la humanidad futura tendrá que lidiar durante milenios. Por tanto, ahí hay una responsabilidad moral con nuestros herederos. Así, estos temas pertenecen al campo o reino de la sabiduría, es decir, a aquel tipo de valores que afectan a la consideración suprema del valor no medial de los otros, por tanto, a sus derechos y dignidad. Y ahí estamos todos. Normalmente los problemas que se tocan en este tercer nivel afectan a todas las personas, sean de la cultura que sean. Por tanto, ya sólo se dan en un marco intercultural.

La interculturalidad es entonces, no un cajón de sastre en el que todo cabe, sino la constitución de un espacio moral (Graciano González 2002 y 2008) en el que el otro es puesto ante todo como una persona con la plenitud de sus derechos, sólo a partir de los cuales podemos dialogar en igualdad de condiciones sobre las preferencias y los intereses sociales. Así, en el terreno técnico no hay interculturalidad sino eficacia de los conocimientos y prácticas. En el terreno práctico, los modos en que en cada cultura se produce la satisfacción de las necesidades de las personas o la organización de su vida, siempre desde el respecto a todos los miembros de su grupo, tampoco hay más interculturalidad que la mencionada del intercambio entre miembros de varias culturas. Donde es necesario sistematizar y pensar a fondo la interculturalidad en los terrenos de los valores morales que incidan en la convivencia y en las decisiones sobre el mundo social y el entorno, en lo que podríamos decir que configura la imagen del ser humano que debe dirigir globalmente la organización de la vida. Y es ahí donde la filosofía es la competente, porque sólo ella está destinada a discutir esos temas, más allá de las ciencias, naturales y humanas, que, como decía Husserl, tienen la primera palabra pero no la última (Husserl 1995, p. 174). 


\section{Referencias bibliográficas:}

Boas, Franz (1911): The Mind of Primitive Man. Nueva York, Macmillan Company. Numerosas ediciones. Trad. al español de la $3^{\text {a }}$ edición inglesa corregida (abril de 1943), por S. W. de Ferdkin, Cuestiones fundamentales de Antropología cultural. con un "Estudio preliminar" de Abraham Monk. Editorial Solar/Librería Hachette, Buenos Aires 1964.

Díaz Álvarez, Jesús M. (2012): "Más allá del racionalismo y el relativismo. Algunas consideraciones sobre la teoría de la perspectiva en El tema de nuestro tiempo", en López/Díaz 2012, pp. 109-128.

González Rodríguez-Arnaiz, Graciano R. (2002): "La interculturalidad como categoría moral”, en Vida Nueva, n²321, 23-30.

- (2008): Interculturalidad y convivencia: el giro intercultural de la filosofia, Madrid, Biblioteca Nueva.

González Esteban, Elsa (2007): "Una lectura actualizada de la ética aristotélica. "La mirada de Martha Nussbaum", Quaderns de fi losofi a i ciència, 37, pp. 91-100

Husserl, Edmund (1987): Aufsätze und Vorträge (1911-1921). Mit ergänzenden Texten herausgegeben von Thomas Nenon und Hans Rainer Sepp. Hua XXV. Incluye el texto "Fichtes Menschenideal (drei Vorlesungen)", pp. 267-293. De este texto hay trad. al inglés de J. G. Hart, "Fichte's ideal of humanity [Three Lectures]", en Husserl Studies, 12, 1995, pp. 111-133.

- (1989):. Aufsätze und Vorträge (1922-1937). Mit ergänzenden Texten, herausgegeben von Thomas Nenon und Hans Rainer Sepp. Hua XXVII. Incluye el texto «Fünf Aufsätze über Erneuerung» (Los artículos sobre "Renovación” para la Revista Kaizo): pp. 3-113, ver Husserl 2002.

- (2002) Renovación del hombre y la cultura. Cinco ensayos. Introducción de G. Hoyos Vásquez. Traducción de A. Serrano de Haro, Barcelona, Anthropos/Universidad Metropolitana de México,

- (1995): "Carta a Lévy-Bruhl", ER, Revista de Filosofía, 19, año X, pp. 171-175. Trad. de J. San Martín.

Iribarne, Julia V. (1987): La intersubjetividad en Husserl. Bosquejo de una teoría. Tomo I, Buenos Aires, Carlos Lohlé.

- (2007): De la ética a la metafísica. En la perspectiva del pensamiento de Edmund Husserl. Bogotá, Universidad Pedagógica Nacional.

Kirk, G. S. y Raven, J. E. (1969): Los filósofos presocráticos. Historia crítica con selección de textos. Versión española de Jesús García Hernández, Madrid, Editorial Gredos.

López, Mª Carmen (2012): "Universalidad existencial (M. Merleau-Ponty) frente al relativismo cultural (C. Lévi-Strauss)”, en López/Díaz 2012, pp. 19- 108. 
López, Ma Carmen y Díaz Álvarez, Jesús M. (2012): Racionalidad y relativismo. En el laberinto de la diversidad, Madrid, Biblioteca Nueva.

Mall, Ram Adhar y Hülsmann, Heinz (1989): Die drei Geburtsorte der Philosophie: China - Indien - Europa. Bonn, Bouvier Verlag.

Nussbaum, M. C. (1992): "Human Functioning and Social Justice: In Defense of Aristotelian Essentialism”, Political Theory, vol. 20 (2), pp. 202-246.

San Martín, Javier (1985): La antropología, ciencia humana, ciencia crítica. Barcelona, Editorial Montesinos. Tercera edición, 2001.

- (1995): "Husserl y la antropología cultural. Comentario a La carta de Husserl a Lévy-Bruhl”, ER, Revista de Filosofía, 19, año X, pp. 177-204.

- (1997a) : Fenomenología y antropología. Buenos Aires, Almagesto. $2^{\mathrm{a}}$ Edición, Buenos Aires/Madrid, Lector/UNED, 2005.

- (1997b): "La philosophie de l'histoire chez Fink et Husserl ", en N. Depraz y M. Richir (ed.), Eugen Fink. Actes du Colloque de Cerisy-la-Salle 2330 juillet 1994, Rodopi Elementa, pp. 231-244, 1997. El texto, traducido, está recogido como capítulo IV de mi libro Para una filosofía de europa. Ensayos de fenomenología de la historia, Madrid, UNED/Biblioteca Nueva, 2007.

- (1999): Teoría de la cultura. Madrid, Editorial Síntesis.

- (2006): "The Life-World: What is Common and What is Different", en Penas, Beatriz y López Sáenz, $\mathrm{M}^{\mathrm{a}}$ Carmen (eds.), Interculturalism. Between Identity and Diversity. Peter Lang, Berna, pp. 63-79. Trad. "Mundo de la vida: lo común y lo diferente", en, Paradojas de la interculturalidad. Filosofía, lenguaje y discurso. Madrid, Editorial Biblioteca Nueva, pp. 61-76. 2008.

- (2012): "La planetarización o globalización, nueva dimensión de la multiculturalidad”, en López/Díaz, 2012, pp. 233-268.

- (2013): Antropología filosófica I. De la Antropología científica a la filosófica, Madrid, UNED. Madrid, UNED.

- (2015): Antropología filosófica II. Vida humana, persona y cultura,

- (2015): "La interculturalidad como génesis de la filosofía. Comentario al antepenúltimo texto de Husserl", en Joan Bautista Llinares y Javier San Martín (coord.) Actas del I Congreso internacional de la Red española de Filosofía. Volumen II. Sección temática 1. Antropología filosófica y teoría de la cultura, vol. Red Española de Filosofía (REF), http://redfilosofia.es, Publicacions de la Universitat de València (PUV), pp. pp. 71-82. 
